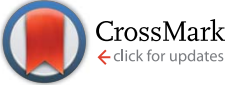

Cite this: RSC Adv., 2017, 7, 7914

\title{
Inhibitory effect of a genistein derivative on pigmentation of guinea pig skin
}

\begin{abstract}
Quancheng Zhou, ${ }^{\text {ab }}$ Chuanxing Feng ${ }^{\mathrm{b}}$ and Zheng Ruan*a
In this study, to improve the properties of genistein, a genistein derivative (GD) was designed and synthesized by combining it with 1-benzylpiperazine. Then, the effects of GD on skin pigmentation in guinea pigs induced by ultraviolet B (UVB) radiation were studied. The guinea pigs were divided into a model group and a GD group. The GD group was coated with different doses of GD for 30 days. Biopsy specimens were taken from the treated skin. Tyrosinase (TYR), malondialdehyde (MDA), superoxide dismutase (SOD) and mammalian target of rapamycin (mTOR), as well as AMP-activated protein kinase (AMPK), were detected according to the commercial kits' protocols. The distribution and secretion of melanin were assayed by hematoxylin-eosin (HE) stain and Fontana-Masson stain methods. The positive staining index of cyclooxygenase-2 (COX-2) and AMPK was detected using an immunohistochemical technique $(\mathrm{IHC})$. UVB radiation resulted in a marked increase in the content of TYR, mTOR and MDA, and the expression of COX-2, and promoted pigmentation in guinea pig skins, but decreased the SOD and AMPK levels. The effect of GD was similar to that of arbutin and could reverse these changes and the effect of GD was the best when its concentration was $2 \%$. The SOD and AMPK levels were increased by approximately $46 \%$ and $40 \%$, meanwhile, the TYR, mTOR and MDA levels and COX-2 expression were reduced by approximately $30 \%, 34 \%, 29 \%$ and $30 \%$, respectively. This study shows that GD can be used in the treatment of UVB-induced pigmentation in guinea pigs.
\end{abstract}

Received 21st November 2016 Accepted 13th January 2017

DOI: 10.1039/c6ra27106e

www.rsc.org/advances and so on..$^{5-7}$ 1-Benzylpiperazine has antioxidant and antiinflammatory activity, and the piperazine moiety has a synergistic effect. ${ }^{8}$ Genistein and resveratrol $\left(1 \mathrm{mmol} \mathrm{L}^{-1}\right)$ effectively abolish melanin synthesis, and convert to orobol and piceatannol, respectively. These results support the strong potential of tyrosinase as a monooxygenase for regio-selective $o$-hydroxylation of various monophenol compounds. ${ }^{9}$ Genistein can reduce the quantity of melanin and the degree of erythema in direct proportion to the number of days of treatment. ${ }^{\mathbf{1 0}}$ Genistein targets TYR directly or indirectly and also induces NRF2 (nuclear factor erythroid 2 [NF-E2]-related factor 2) in different cellular backgrounds for invoking a protective antioxidant mechanism. ${ }^{11}$

Despite the important roles attributed to genistein in nutrition, the effect and mechanism of genistein supplementation on pigmentation or melanin synthesis has received little attention. The objective of the present study is to clarify the mechanisms of genistein derivative (GD) on skin pigmentation in a guinea pig model.

\section{Materials and methods}

\section{Chemicals}

Genistein, 1-benzylpiperazine, 95\% ethanol, 37\% formaldehyde, ethyl acetate, chloroform, dimethyl sulfoxide (DMSO), sodium sulfide and arbutin were purchased from Tianjin 
Zhiyuan Chemical Reagent Co. Ltd (Tianjin, China). Guinea pig MDA ELISA Kit, Guinea pig SOD ELISA Kit, Guinea pig TYR ELISA Kit, Guinea pig mTOR ELISA Kit and Guinea pig AMPK ELISA Kit were purchased from Shanghai Lanpai Biological Technology Co. Ltd.

\section{Synthesis of GD}

$1 \mathrm{mmol}$ of genistein $(270 \mathrm{mg}), 25 \mathrm{~mL}$ of $95 \%$ ethanol, and 0.08 $\mathrm{mL}$ of $37 \%$ formaldehyde were added to a $50 \mathrm{~mL}$ round-bottom flask. The reaction solution was heated to about $55^{\circ} \mathrm{C}$, and after the reaction solution was clarified, $1 \mathrm{mmol}$ of 1-benzylpiperazine $(174 \mu \mathrm{L})$ was added, then the reaction solution was stirred at room temperature for about $48 \mathrm{~h}$. When the solid separated out, the solution was filtered and dried to get a solid powder. The solid powder was collected and purified by recrystallization (chloroform : 95\% ethanol $=2: 1$ ).

The yield of GD obtained from the reaction is $58 \%$. Its melting range is $166.4{ }^{\circ} \mathrm{C}$ to $167.5{ }^{\circ} \mathrm{C}$, as measured by the melting point apparatus, and its structure (Fig. 1Ad) was characterized using elemental analysis and ${ }^{1} \mathrm{H}$ nuclear magnetic resonance spectrometry $\left({ }^{1} \mathrm{H}-\mathrm{NMR}\right)$. The result of the ${ }^{1} \mathrm{H}-\mathrm{NMR}$ (DMSO- $\mathrm{d}_{6}, 400 \mathrm{MHz}$ ) (Fig. 1B and C) is 1.25-1.35 (s, 1H), 3.55-3.65 (m, 3H), 3.75-3.85 (d, 2H), 3.91-3.98 (d, 2H), 6.316.39 (s, 1H), 6.89-6.95 (d, 2H), 7.33-7.45 (m, 6H), 7.83-7.89 (s, $1 \mathrm{H})$, and $12.85-13.31(\mathrm{~s}, 1 \mathrm{H})$. The molecular formula of GD is $\mathrm{C}_{27} \mathrm{H}_{28} \mathrm{~N}_{2} \mathrm{O}_{5}$ (C, 70.42; H, 6.13; N, 6.08), and the molecular weight is 460.52 .

\section{Experimental animals}

White guinea pigs (350-380 g) were supplied by Shandong Lukang Animal Pharmaceutical Co. Ltd. The production license number of the company is Scxk Lu 2013001.

\section{UVB irradiation-induced pigmentation in white guinea pigs}

All experimental procedures were approved by the Institutional Animal Care and Use Committee (IACUC) of China, and followed the institutional guidelines for animal welfare and experimental conduct (Permit Number: IACUC2015-011). White guinea pigs were housed in cages under standard experimental conditions $\left(22 \pm 1{ }^{\circ} \mathrm{C} ; 55 \pm 5 \%\right.$ humidity; $12 \mathrm{~h}$ light and $12 \mathrm{~h}$ dark cycle) and were maintained on a standard diet with freely available water. After a week of adaptation, the dorsal hair of the white guinea pigs was shaved off. Then, six separate square $(1 \mathrm{~cm} \times 1 \mathrm{~cm})$ dorsal areas on each guinea pig were exposed to $83 \mathrm{~mJ} \mathrm{~cm}{ }^{-2}$ of UVB light with a distance of $10 \mathrm{~cm}$ between the light source (321 nm, Philips, China) and the target skin for 24 min per day for up to 5 days..$^{12,13}$

\section{External inunctum}

Guinea pigs (21) were used for all experiments. Each guinea pig had six separate squares $(1 \mathrm{~cm} \times 1 \mathrm{~cm})$ on its back. These squares were the high dose group, middle dose group, low dose group, model group, DMSO group and arbutin group. GD and arbutin were dissolved in DMSO to prepare $1 \%, 2 \%$, and $4 \%$ GD solutions and $2 \%$ arbutin solution, respectively. Starting from
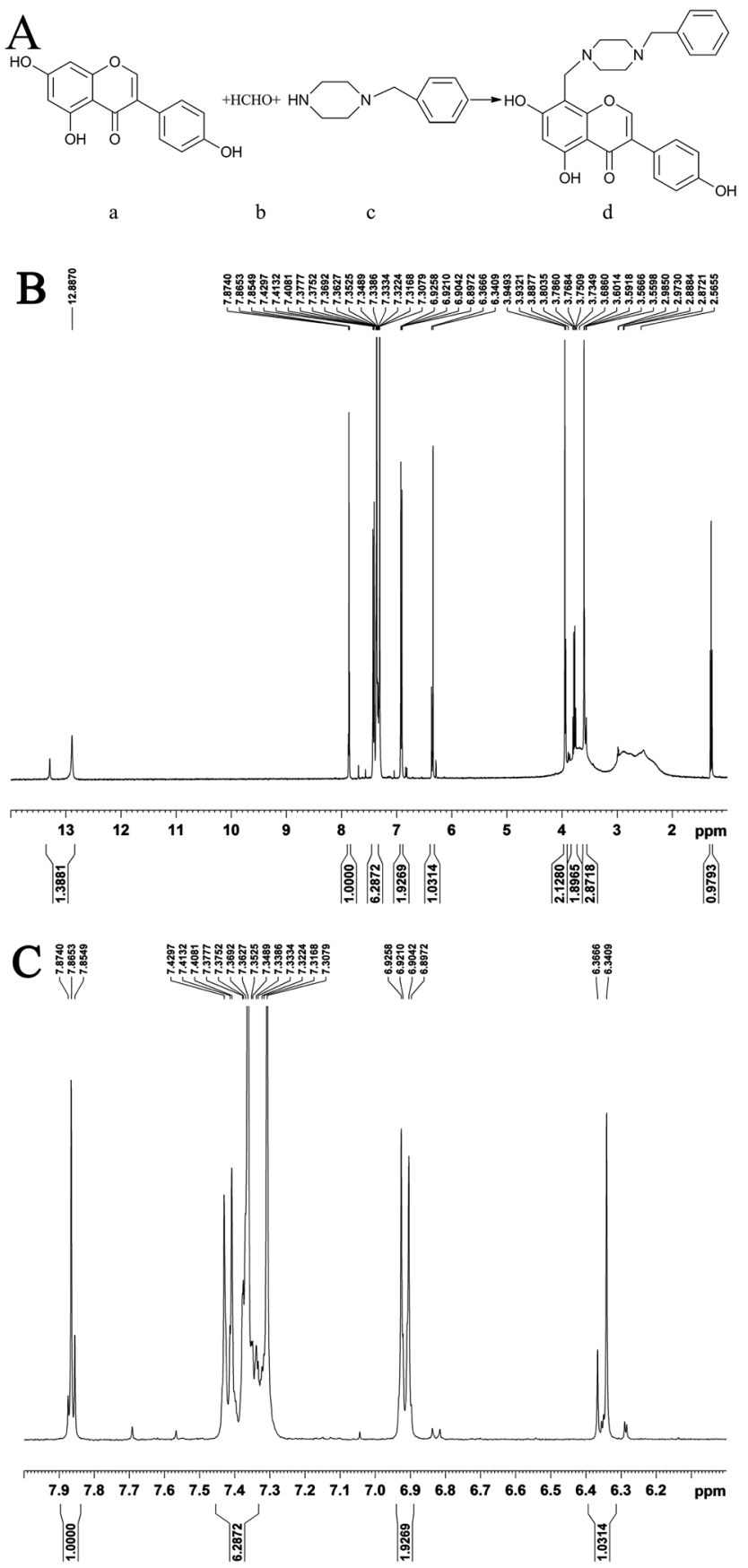

Fig. 1 The synthetic route of GD ((Aa) genistein, (Ab) formaldehyde, (Ac) 1-benzylpiperazine and (Ad) GD). (B and C) ${ }^{1} \mathrm{H}$-NMR spectra of GD in DMSO- $d_{6}(400 \mathrm{MHz})$.

the day after the last dose of UVB irradiation, each sample solution was applied topically with a pipette to the separate shaved areas once a day for 30 days. The high dose group, medium dose group and low dose group were coated with 250 $\mu \mathrm{L}$ of $4 \%, 2 \%$ and $1 \%$ GD solution, respectively. The model group was only irradiated with UVB and not coated with anything. The DMSO group was coated with $250 \mu \mathrm{L}$ of DMSO and the arbutin group was coated with $250 \mu \mathrm{L}$ of $2 \%$ arbutin solution. 


\section{Growth performance and skin color}

The general state of the guinea pigs, including their diet and the color of the skin on the back of the guinea pigs, was observed every day.

\section{Histopathological analysis}

The guinea pigs were suffocated to death by ethyl ether on the 31st day. The six separate squares of the dorsal skin tissues were surgically removed and incubated in $12 \%$ formalin to fix them. The skins were made into conventional paraffin-embedded sections. The slides were dried after an anti-shedding treatment. HE stain and Fontana-Masson stain were used to show the melanin granules in the epidermis, and the distribution of melanin in the skin was observed. ${ }^{\mathbf{1 4}}$

\section{Determination of MDA, SOD, TYR, MTOR and AMPK}

The dorsal glabrous skins of the guinea pigs were cleaned with saline $\left(4^{\circ} \mathrm{C}\right)$. The back skin tissue $(0.5 \mathrm{~g})$ of each guinea pig was placed in a $4 \mathrm{~mL}$ centrifuge tube to which was added $2.0 \mathrm{~mL}$ of pre-cooled saline in advance. Then, the skin tissue was homogenized 2 times (10 s/time) using a XHF-1 high speed disperser (10 $000 \mathrm{rpm})$. After mixing evenly, the supernatant was collected using a TDZ5-WS centrifuge (3500 rpm; $15 \mathrm{~min} ; 4$ $\left.{ }^{\circ} \mathrm{C}\right)$. After centrifugation, the supernatant was preprocessed using the commercial kits' protocols. Finally, the optical densities of the supernatant were measured with a RT-6100 microplate reader at $450 \mathrm{~nm}$, and then compared to standard curves to get the concentrations of MDA, SOD, TYR, mTOR and AMPK in the sample.

\section{Immunohistochemistry for AMPK and COX-2}

The expression of AMPK and COX-2 in the dorsal skin tissues was evaluated using an immunohistochemical technique (IHC). The slides were deparaffinized, hydrated, immersed in $0.3 \%$ $\mathrm{H}_{2} \mathrm{O}_{2}$ (30 min), covered with normal $6 \%$ goat serum (10 min), incubated with primary antibodies (Boster Biological Technology, Wuhan, China) $\left(2 \mathrm{~h} ; 4{ }^{\circ} \mathrm{C}\right)$, reacted with secondary antibodies ( $30 \mathrm{~min} ; 37^{\circ} \mathrm{C}$ ), stained with 3,3-diaminobenzidine (DAB) (Sigma, St Louis, MO, USA), and counterstained. Finally, the slides were visualized under a light microscope at $200 \times$ magnification. The index of positive staining was expressed as the integral optical density (IOD) processed by an Image ProPlus 6.0. ${ }^{15}$

\section{Statistical analysis}

Statistical analysis was performed using SPSS 19.0 statistical software. All data were expressed as mean \pm standard error of mean (S.E.M.). The differences among the groups were analyzed by a one-way analysis of variance (ANOVA) followed by Dunnett's test. A level of $p<0.05$ was considered significant.

\section{Results and discussion}

\section{General situation}

When the dose of UVB irradiation reached $6000 \mathrm{~mJ} \mathrm{~cm} \mathrm{~cm}^{-2}$, the guinea pigs were found to have a loss of appetite and a reduction in the amount of water they were drinking, and their dorsal skin turned red. The next day, the skin began to desquamate. The skin began to show pigmentation when the irradiation dose of UVB was $10000 \mathrm{~mJ} \mathrm{~cm} \mathrm{~cm}^{-2}$.

\section{HE stain analysis}

The HE stain can display the cellular and tissue morphology, but it cannot specifically identify melanin granules, because it is nonspecific. ${ }^{14}$ As shown in Fig. 2, after UVB irradiation, the spinous layer was obviously thickened, the epidermal structure was incomplete, the layer was obscure, the dermal layer was loose, the dermal fibers were degenerated and destructed, the dermal tissue was arranged in disorder and inflammatory cells were infiltrated in it uniformly. However, treatment with GD and arbutin could improve this situation, but the inhibitory effects of GD were not dose-dependent, and the $2 \%$ GD-treated specimens showed a significant result.

\section{Fontana-Masson stain analysis}

The biopsy specimens were stained with Fontana-Masson which can enhance visualisation of melanin granules to assess the content of epidermal melanin after treatment with the different concentrations of GD. From Fig. 3, we could see that the skin pigmentation model of guinea pigs induced by UVB irradiation was successful. The relative density of epidermal melanin was reduced in the specimens treated with arbutin and GD compared to that in the model group. In particular, the $2 \%$ GD-treated specimens showed a significant reduction in melanin content.

\section{Effect of GD on oxidative stress markers}

Superoxide dismutase (SOD) is a biological antioxidant enzyme. It has an antioxidant function and it is the only enzyme that can remove the superoxide anion free radical $\left(\mathrm{O}_{2}{ }^{-\bullet}\right)$ produced by

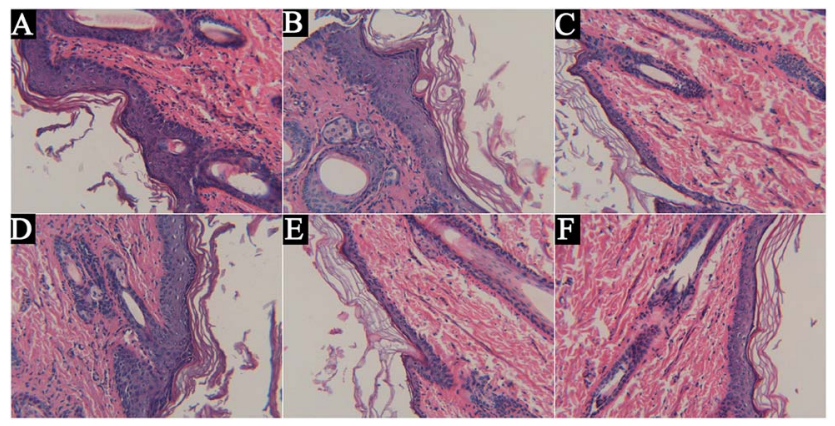

Fig. 2 Effects of GD, arbutin, and DMSO on histopathological changes in UVB-irradiated skin tissues of white guinea pigs. Tissue morphology was visualized with HE stain. (A) Model; (B) DMSO; (C) $2 \%$ arbutin; (D) $1 \%$ GD; (E) 2\% GD; (F) 4\% GD. Magnification is $200 \times$. 


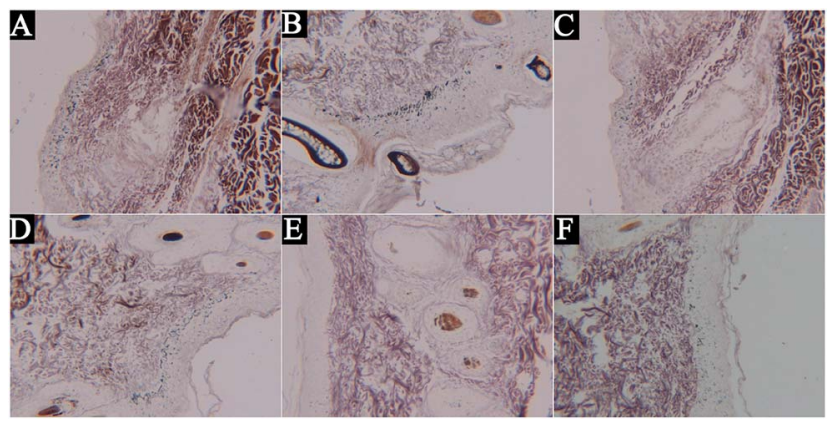

Fig. 3 Effects of GD, arbutin, and DMSO on hyperpigmentation in UVB-irradiated skin tissues of white guinea pigs. Melanin content is visualized with Fontana-Masson stain. (A) Model; (B) DMSO; (C) $2 \%$ arbutin; (D) 1\% GD; (E) 2\% GD; (F) 4\% GD. Magnification is 200×.

biological oxidation in nature. ${ }^{16}$ The antioxidant capacity and the ability in removing free radicals can increase as the level of SOD increases. ${ }^{17}$ Free radicals can cause lipid peroxidation. MDA is a degradation product of lipid peroxides, which can make the proteins crosslink to form pigments. ${ }^{3}$ Therefore, SOD scavenges free radicals to make the level of MDA decrease, which can show that the oxidation degree of the guinea pig skin is decreased.

The results of Fig. 4 show that there was no significant difference between the DMSO group and the model group in the levels of SOD and MDA $(P>0.05)$. This indicated that DMSO had no effect on the levels of MDA and SOD in the guinea pig skin. In the GD group, the level of MDA was lower and the level of SOD was higher than in the model group, and there was a markedly significant difference between the two groups $(P<$ 0.01). In particular, the effect of the middle dose group ( $2 \% \mathrm{GD})$ was best. It can be concluded that the effect of GD is similar to that of arbutin, which can inhibit the skin pigmentation of guinea pigs induced by UVB irradiation.

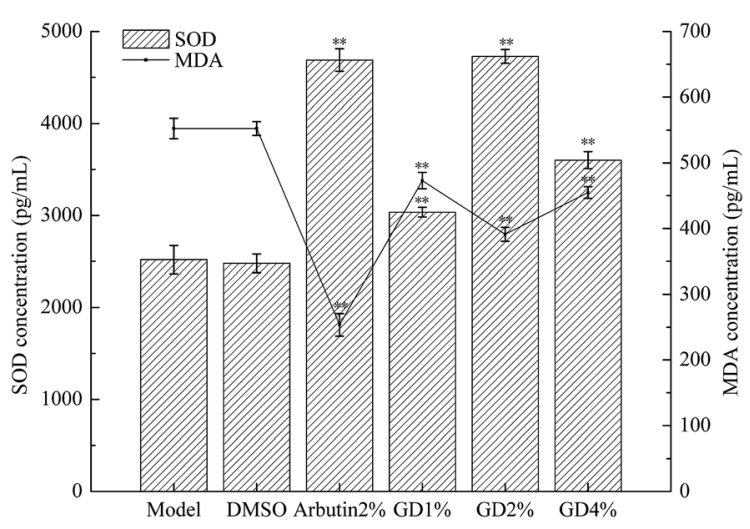

Fig. 4 Effects of GD, arbutin, and DMSO on SOD and MDA concentrations in UVB-irradiated skin tissues of white guinea pigs. The results are the mean \pm S.E.M. One-way ANOVA test followed by Dunn's test, ** indicates that $P<0.01$ between the GD groups and the model group.

\section{Effect of GD on the level of melanogenic enzyme}

Tyrosinase (TYR), which is the main rate-limiting enzyme in the process of the production of melanin, is a kind of polyphenol oxidase containing copper. ${ }^{18}$ Its activity is positively related to the synthesis of melanin. ${ }^{19}$ TYR mainly controls the synthesis of tyrosine (Tyr) which is the main raw material for the formation of melanin. Inhibiting the activity of Tyr can suppress the formation of melanin. ${ }^{20}$

As shown in Fig. 5, compared with the model group, DMSO administration did not achieve statistical significance $(P>0.05)$. GD could significantly reverse the overproduction of TYR $(P<$ 0.01 ) and the effect of $2 \%$ GD was approximately the same as that of the arbutin which could decrease the level of TYR to inhibit the synthesis of tyrosine.

\section{Effect of GD on the levels of AMPK and mTOR}

AMP-activated protein kinase (AMPK) and the mammalian target of rapamycin (mTOR) are serine/threonine protein kinases. Moreover, the signal pathways of AMPK and mTOR are associated with each other, and the increase of AMPK concentration can inhibit mTOR and its effector. ${ }^{21}$ Through phosphorylation of S6K1 and 4E-BP1, on the one hand, mTOR can regulate the proliferation of melanocytes, while on the other hand, it can regulate and promote the synthesis of tyrosine. ${ }^{22}$ Therefore AMPK plays an important role in inhibiting the synthesis of tyrosine and the proliferation of melanocytes by regulating the mTOR pathway. ${ }^{23}$

Fig. 6 indicates that DMSO had no effect on the levels of AMPK and mTOR in guinea pig skin. Pretreatment with GD resulted in a noticeable increase in the AMPK level (Fig. 6A) compared with that in the model group $(P<0.01)$. The expression of AMPK, tested by immunohistochemical staining (Fig. 6B and $\mathrm{C}$ ), also increased. In addition, potent reduction of the mTOR (Fig. 6A) level was determined after GD administration. GD at the dose of $2 \%$ showed a potent effect that was similar to that of arbutin which could inhibit the synthesis of tyrosine and the growth and reproduction of melanocytes.

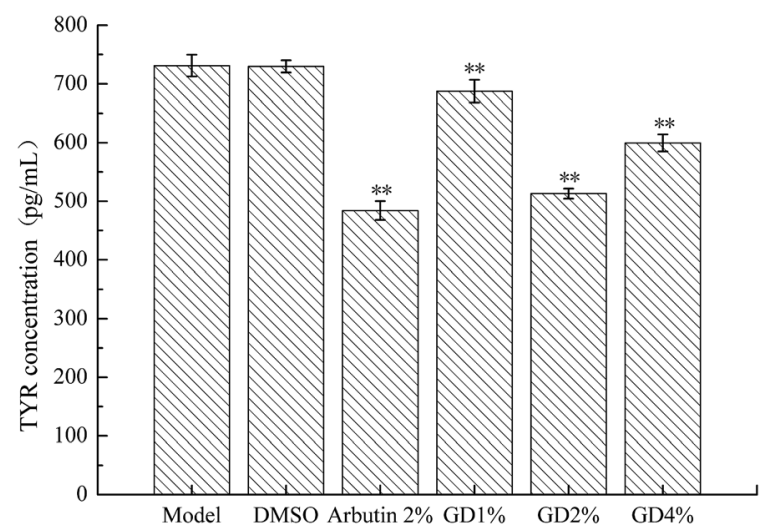

Fig. 5 Effects of GD, arbutin, and DMSO on TYR concentrations in UVB-irradiated skin tissues of white guinea pigs. The results are the mean \pm S.E.M. One-way ANOVA test followed by Dunn's test, ** indicates that $P<0.01$ between the GD groups and the model group. 
A

C
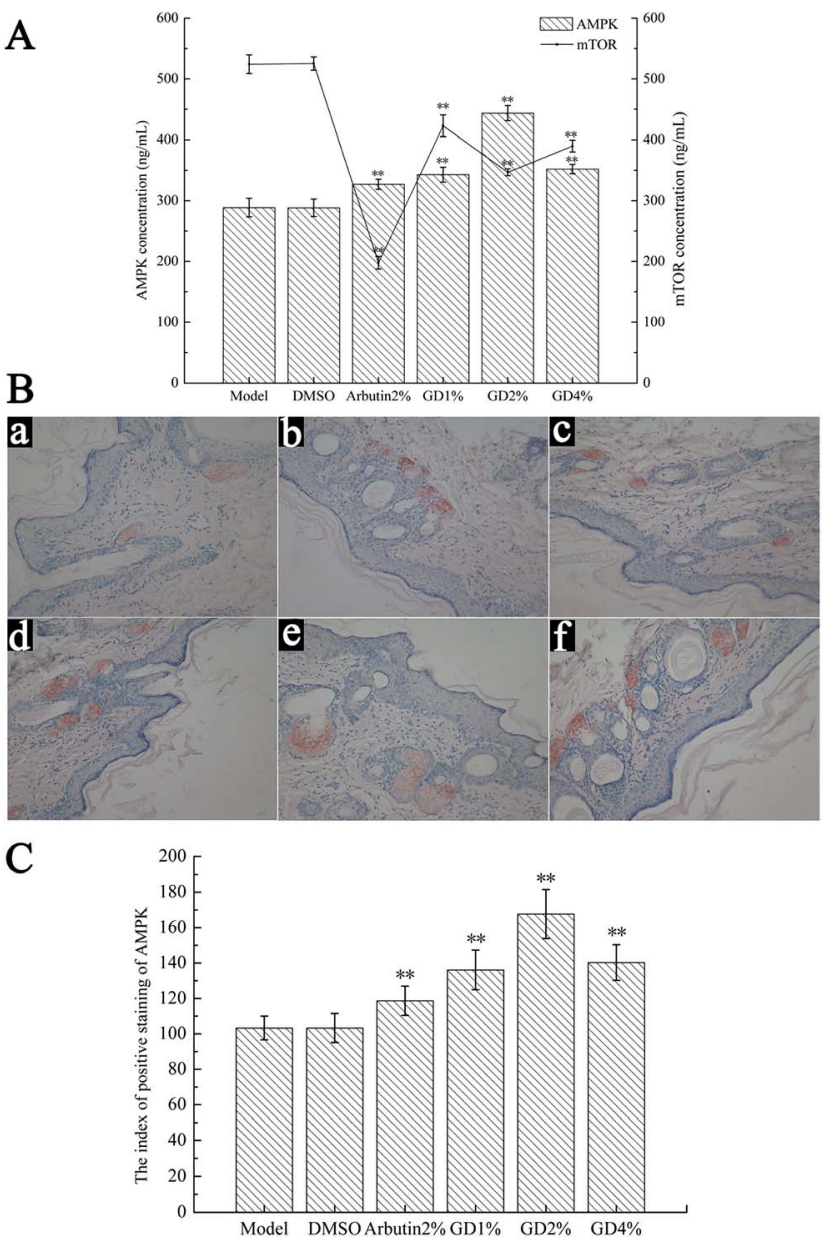

Fig. 6 (A) Effects of GD, arbutin, and DMSO on AMPK and mTOR concentrations in skin tissues. (B) The expression of AMPK in skin tissues ((a) model; (b) DMSO; (c) 2\% arbutin; (d) 1\% GD; (e) 2\% GD; (f) 4\% GD). (C) The index of positive staining of AMPK in each group. Magnification is $200 \times$. The results are the mean \pm S.E.M. One-way ANOVA test followed by Dunn's test, ** indicates that $P<0.01$ between the GD groups and model group.

\section{Effect of GD on the expression of COX-2}

Cyclooxygenase-2 (COX-2) is the inducible form of the COX enzymes, and it can catalyze prostaglandin biosynthesis from arachidonic acid. ${ }^{24}$ UVB irradiation induces the production of COX-2 to increase the PGE-2 level which is the factor of melanin synthesis, and then promotes the synthesis of melanin..$^{25}$ Some studies have found that AMPK is the "cellular energy regulator", and COX-2 can accept the energy signal, so there is an AMPKCOX-2 signal pathway between AMPK and COX-2. ${ }^{26}$

The expression of COX-2 in the skin tissues of white guinea pigs was tested using immunohistochemical staining. Fig. 7A and $\mathrm{B}$ indicate that UVB irradiation increased the expression of COX-2 in the skin tissues of white guinea pigs. Compared with the model group, GD $(1 \%, 2 \%$ and $4 \%)$ and arbutin $(2 \%)$ could significantly reverse the overproduction of COX-2. GD at doses of $1 \%, 2 \%$, and $4 \%$ achieved statistical significance when compared with the model group $(P>0.05)$. In particular, an

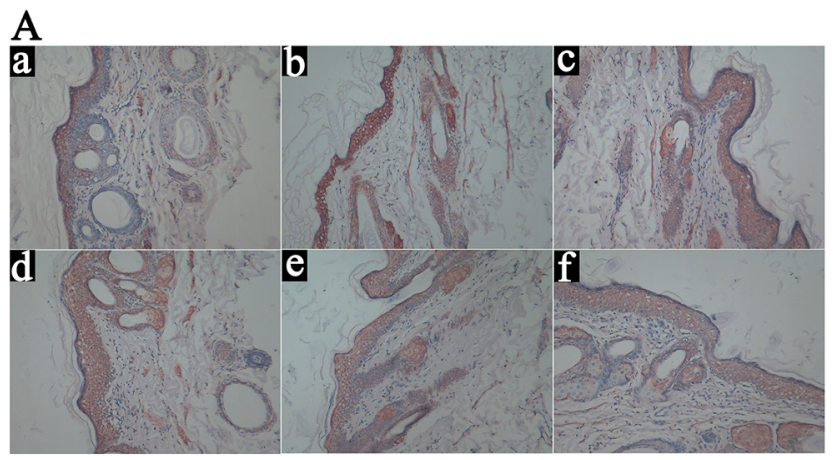

B

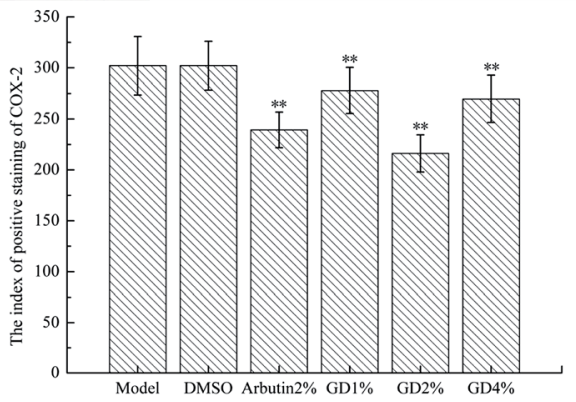

Fig. 7 Effects of GD, arbutin, and DMSO on COX-2 expression in skin tissues. (Aa) Model; (Ab) DMSO; (Ac) 2\% arbutin; (Ad) 1\% GD; (Ae) 2\% GD; (Af) $4 \%$ GD. (B) The index of positive staining of COX-2 in each group. Magnification is $200 x$. The results are the mean \pm S.E.M. Oneway ANOVA test followed by Dunn's test, $* *$ indicates that $P<0.01$ between the GD groups and model group.

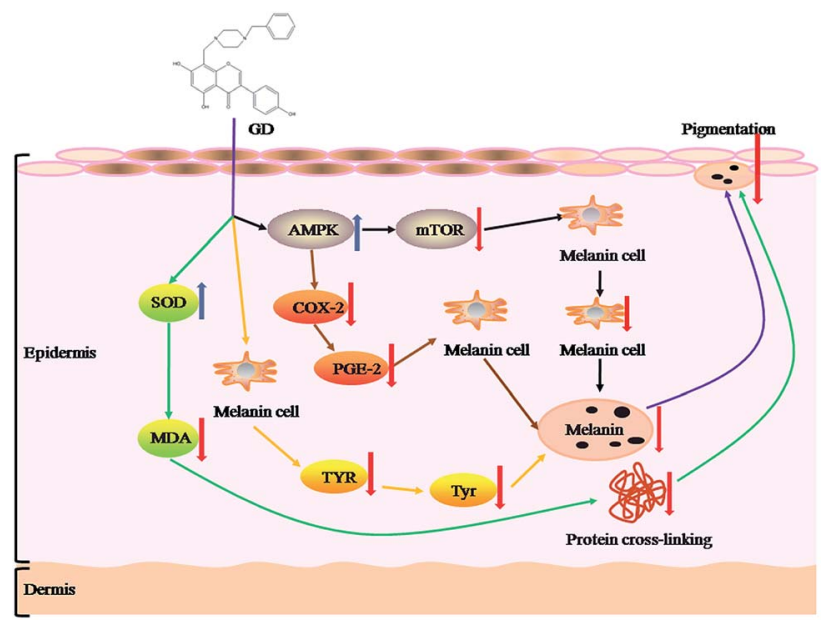

Fig. 8 The mechanisms of the inhibitory effects of GD against UVBinduced pigmentation in guinea pig skin.

approximately $30 \%$ reduction of COX-2 expression in skin tissues was determined after $2 \%$ GD administration.

\section{Conclusions}

This study demonstrated the inhibitory effect of GD on pigmentation in a guinea pig model from pathology and physiological activity. From the pathological histology section, the skin pigmentation was obviously reduced after GD treatment. 
From the physiological activity, GD inhibited the formation of melanin by four pathways (Fig. 8). First, GD inhibited the formation of melanin by inhibiting the production of TYR. Second, GD increased the level of SOD to improve the antioxidant capacity of the skin, so that the ability of the skin to remove MDA was enhanced, and reduced protein cross-linking to form melanin. Third, GD inhibited the production of mTOR, which is a melanoma cell growth regulator, by promoting AMPK. The number of melanoma cells was reduced, which inhibited the synthesis of melanin. Last, GD decreased the expression of COX-2 by the AMPK-COX-2 signal pathway. The decrease of COX-2 expression caused the decrease of the PGE-2 level which was the factor of melanin synthesis. It also demonstrated the inhibitory effect of GD on pigmentation.

Arbutin is the drug known to treat skin pigmentation. The results of this study found that the inhibitory effect of $2 \%$ GD on skin pigmentation is better than that of arbutin. In conclusion, this study provides a new method for the treatment of skin pigmentation.

\section{Acknowledgements}

This study was supported by the Open Project Program (SKLFKF-201414) of State Key Laboratory of Food Science and Technology, Nanchang University.

\section{References}

1 J. Fang, W. Y. Zhu and M. H. Zhang, Am. J. Clin. Dermatol., 2007, 36, 141-144.

2 A. K. Gupta, M. D. Gover, K. Nouri and S. Taylor, J. Am. Acad. Dermatol., 2007, 55, 1048-1065.

3 L. Cao and Y. Dai, World Clin. Drugs, 2007, 28, 526-531.

4 J. Fang, S. J. Du and Y. L. Jin, J. Hyg. Res., 2009, 38, 111-113.

5 L. Liu, X. Li, F. Liu and X. W. Deng, Chin. J. Arterioscler., 2008, 16, 928-932.

6 C. L. Liu, Z. Q. Li, H. X. Sun and C. S. Li, Soybean Sci., 2008, 27, 693-696.

7 C. M. Rassi, M. Lieberherr and G. Chaumaz, J. Bone Miner. Res., 2002, 17, 630-638.
8 J. L. Cai, Y. H. Lu, L. L. Gan, Y. Y. Zhang and C. H. Zhou, Chin. J. Antibiot., 2009, 34, 454-462.

9 S. H. Lee, K. Baek, J. E. Lee and B. G. Kim, Biotechnol. Bioeng., 2016, 113, 735-743.

10 C. Danciu, F. Borcan, F. Bojin, I. Zupko and C. Dehelean, Nat. Prod. Commun., 2013, 8, 343-346.

11 L. S. Feng and M. Frank, Mol. Nutr. Food Res., 2016, 60, 12641274.

12 G. Imokawa, M. Miyagishi and Y. Yada, J. Invest. Dermatol., 1995, 105, 32-37.

13 Y. Yoshida, A. Hachiya, P. Sriwiriyanont, A. Ohuchi, T. Kitahara, Y. Takema, M. O. Visscher and R. E. Boissy, FASEB J., 2007, 21, 2829-2839.

14 K. T. Park, J. K. Kim, D. Hwang, Y. Yoo and Y. H. Lim, Food Chem. Toxicol., 2011, 49, 3038-3045.

15 H. Zheng, Y. L. Chen, J. Z. Zhang, L. Wang, Z. X. Jin, H. H. Huang, S. L. Man and W. Y. Gao, Chem.-Biol. Interact., 2016, 250, 68-77.

16 Y. H. Hu and J. K. Niu, Biology Teaching, 2005, 30, 2-3.

17 A. D. Lin and A. Mannikarottu, Mol. Cell. Biochem., 2007, 296, 11-16.

18 A. Sánchez-ferrer, J. N. Rodríguez-Lopez, F. García-Cánovas and F. García-Carmona, Biochim. Biophys. Acta, Protein Struct. Mol. Enzymol., 1995, 1247, 1-11.

19 H. M. Zhang, J. Gen and Q. C. Zhou, J. Chin. Cereals Oils Assoc., 2013, 28, 96-97.

20 Q. X. Chen and K. K. Song, J. Xiamen Univ., Nat. Sci., 2009, 45, 733-734.

21 Q. Y. Fu and Y. Q. Gao, Chin. Sci. Bull., 2005, 17, 149.

22 L. J. Chen and D. H. Shi, J. Mod. Oncol., 2012, 20, 2435-2436.

23 F. Cai, J. L. Su, P. Ge, Q. Ai and L. Zhang, Chemistry for Life, 2015, 35, 26-27.

24 Y. K. Lee, S. Y. Park, Y. M. Kim, W. S. Lee and O. J. Park, Exp. Mol. Med., 2009, 41, 201-207.

25 K. Kazue, M. H. Hiroko, T. Kiyoji, E. Naomi, I. Takashi, U. Yoshihiro and H. Takeshi, J. Invest. Dermatol., 2000, 114, 241-246.

26 J. Zhang and B. G. Tim, Mol. Carcinog., 2008, 47, 974-983. 